\title{
Interaction between a Normal Shock Wave and a Turbulent Boundary Layer at High Transonic Speeds.
}

\section{Part I: Pressure Distribution}

By A. F. Messiter, Dept of Aerospace Engineering, The University of Michigan, Ann Arbor, Michigan, USA

\section{Introduction}

In several recent studies, asymptotic methods have been used successfully for the derivation of rational approximations which describe the interaction of a turbulent boundary layer and a weak, stationary, normal shock wave. It appears that correct limiting forms of the equations can be determined, that numerical or analytical solutions to these equations are obtained easily enough to be of practical interest, and that numerical accuracy may be adequate for important parameter ranges. In the limiting case to be considered here, still for an unseparated boundary layer, the shock wave extends close to the wall, the upstream influence is small, and analytical solutions can be obtained for most of the flow field. Pressure distributions are derived in Part I; the wall shear stress and the possibility of predicting separation will be discussed in Part II.

In many transonic flows of interest, there occurs a shock wave which, in an inviscid-flow approximation, is normal to a solid boundary, at values of the Reynolds number large enough that the boundary layer along the wall is fully turbulent. Since the strength of the shock wave must decrease to zero in the supersonic part of the boundary layer, there can be no discontinuity in the pressure at the wall. It is observed that the shock wave becomes slightly curved and is displaced slightly in the upstream direction. As the Mach number upstream is increased, still below the value required for separation, the shock wave extends further into the boundary layer; experimental results $[1,2]$ show an initially rapid rise in the wall pressure, followed by a gradual decrease in the pressure gradient over a distance several times larger than the boundary-layer thickness.

Asymptotic descriptions of these flows, in the limit of infinite Reynolds number, have been discussed in References [3] through [10]; in particular, Reference [8] contains the first steps of the present work. In each of these studies, the representation of the undisturbed boundary layer in terms of a velocity-defect layer and a wall layer [11-13] is regarded as providing an asymptotic description as the Reynolds number tends to infinity [14-18]. The pressure gradient in the boundary layer is large near the 
shock wave, and consequently the forces resulting from changes in the Reynolds stresses are of higher order than terms retained, in most of the boundary layer. Thus, as for laminar flow [19-21], an asymptotic description of the changes in the mean flow can be obtained with the use of inviscid-flow equations for most of the boundary layer.

The form of the velocity profile, however, implies two important differences from the laminar-flow case. First, for an unseparated turbulent boundary layer the wall layer is extremely thin, and the displacement effect resulting from deceleration of fluid close to the wall remains very small, even in a large pressure gradient. Thus, if the undisturbed velocity profile is known outside the wall layer, an approximation to the pressure can be found without knowledge of the flow details near the wall and therefore without any further assumption about the nature of the turbulent stresses. Second, for a slightly supersonic external flow the sonic line is located at an arbitrary position (outside the wall layer) in the undisturbed boundary layer, depending on the relative sizes of the nondimensional friction velocity and the nondimensional difference between the fluid velocity and the critical sound speed in the external flow. As the Reynolds number tends to infinity, one can then study three cases, such that the ratio of these parameters tends to infinity, remains constant, or approaches zero.

Adamson and Feo [3] considered an incident oblique shock wave in a flow with velocity only slightly greater than the sound speed, such that the sonic line is located very close to the edge of the boundary layer. The corresponding asymptotic formulation was shown to lead to a local-interaction problem requiring solution of the transonic small-disturbance equations for the local perturbations in the external flow, expressed in appropriately scaled variables. The influence of the boundary layer is represented on this scale through an effective wall boundary condition specifying a linear relationship between the streamline slope and the pressure gradient. Melnik and Grossman [4] studied a normal shock wave having strength, as measured by the nondimensional pressure jump, of the same order as the friction velocity, so that in the limit the sonic line is at an arbitrary location in the boundary layer. Numerical solutions of the transonic small-disturbance equations were obtained for perturbations in the defect portion of the boundary layer and in the neighboring external flow. Changes in the wall layer were also discussed in each of these papers. Melnik and Grossman later $[5,6]$ obtained additional numerical solutions for axisymmetric pipe flow. At higher upstream speeds, which might be characterized as 'high transonic speeds', the shock wave is stronger but the boundary layer can remain unseparated. For this case, a first approximation for the flow perturbations outside the wall layer was given by Adamson and Messiter [8]. The shock-wave strength, although still small, was taken to be large in comparison with the nondimensional friction velocity, so that in the undisturbed boundary layer the distance from the sonic line to the wall is much smaller than the boundary-layer thickness. The corresponding problem has also been discussed for an incident oblique shock wave [7,9]. A brief preliminary description of some of the present results was given in Ref. [10]; a few details have since been modified. 
In the present work, analytical solutions are derived which incorporate additional physical effects as higher-order terms for the case, first discussed rather briefly in Ref. [8], when the sonic line is very close to the wall. The functional form used for the undisturbed velocity profile is described in Section 2, to indicate how various parameters will be calculated for later comparison with experiment. The basic solutions for the pressure distribution are derived in Section 3. In Section 4 corrections are added for flow along a wall having longitudinal curvature and for flow in a circular pipe, and comparisons with available experimental data are shown. It was also found possible, by a more lengthy derivation, to remove the restriction to weak shock waves; for the parameter ranges of greatest practical interest, the numerical results were seen to differ only slightly from the simpler solutions of Section 3, and the longer derivation has therefore been omitted here.

\section{Undisturbed Velocity Profile}

Nondimensional rectangular coordinates $X$ and $Y$ are measured along and normal to the wall, respectively, with $Y=0$ at the wall and $X=0$ at some point on the shock wave, e.g., at the intersection of the shock wave with the edge of the boundary layer as defined below. The reference length is a geometric length such as the length of the boundary layer from a leading edge up to the shock wave. The nondimensional mean-velocity components $U$ and $V$, referred to the critical sound speed in the external flow, are in the $X$ and $Y$ directions respectively, and the term $\overline{\rho^{\prime} V^{\prime}} \mid \rho$ has been included in $V$. Here primes denote fluctuations about the mean, and $\overline{\rho^{\prime} V^{\prime}}$ denotes an average value. The nondimensional mean pressure $P$, density $\rho$, temperature $T$, and viscosity coefficient $\mu$ are referred to the critical values of pressure, density, and temperature, and the corresponding viscosity coefficient, in the flow just outside the boundary layer and ahead of the shock wave. The sum of the nondimensional Reynolds stress and viscous stress, in the boundary-layer approximation, is denoted by $\tau$, and has been made nondimensional with twice the dynamic pressure, in terms of the same reference quantities. For later convenience the friction velocity $u_{\tau}$ is made nondimensional using the external-flow density:

$$
u_{\tau}^{2}=\frac{\tau_{w}}{\rho_{e}}=\frac{1}{2} U_{e}^{2} c_{f}, \quad U_{e}=1+\epsilon
$$

where the subscripts $e$ and $w$ indicate values in the external flow and at the wall, respectively, and $c_{f}$ is the undisturbed value of the skin friction coefficient, referred as usual to the dynamic pressure in the external flow. The nondimensional difference between the fluid velocity and the critical sound speed in the external flow is $\epsilon$, and in the present case $u_{\tau} \ll \epsilon \ll 1$. For simplicity, an adiabatic wall is assumed and the total enthalpy is taken to be uniform. The ratio of specific heats is $\gamma$ and is constant.

As in references cited above, it is assumed that the undisturbed boundary layer can be described asymptotically in terms of a velocity-defect layer and a wall layer. 
The defect layer occupies most of the boundary layer, and its thickness is taken equal to a boundary-layer thickness $\delta$. The velocity differs from the external-flow velocity by an amount of order $u_{\tau}$, the shear stress is $\tau=O\left(u_{\tau}^{2}\right)$, and the layer thickness is $\delta=O\left(u_{\tau}\right)$. The much thinner wall layer has thickness denoted by $\tilde{\delta}$, and the velocity there is small, of order $u_{\tau}$. Coordinates measured in terms of these nondimensional thicknesses are defined by

$$
\begin{array}{ll}
y=\frac{Y}{\delta}, & \delta=O\left(u_{\tau}\right) \\
\tilde{y}=\frac{Y}{\tilde{\delta}}, & \tilde{\delta}=\frac{\mu_{w}}{\mu_{e}}\left(\frac{T_{w}}{T_{e}}\right)^{1 / 2} \frac{U_{e}}{u_{\tau}} \frac{1}{\operatorname{Re}}
\end{array}
$$

where $\tilde{\delta} \ll \delta$, and $\tilde{\delta}$ has been set equal to the ratio of the nondimensional local kinematic viscosity and a friction velocity $u_{\tau}\left(T_{w} / T_{e}\right)^{1 / 2}=\left(\tau_{w} / \rho_{w}\right)^{1 / 2}$ based on the density at the wall. The Reynolds number $\mathrm{Re}$ is based on the geometric reference length and the undisturbed external-flow velocity and kinematic viscosity; all parameters are understood to be evaluated immediately upstream of the shock wave.

The velocity $U_{u}$ in the undisturbed boundary layer just ahead of the shock wave is expressed in the defect layer in terms of $y$ and in the wall layer in terms of $\tilde{y}$, as follows:

$$
\begin{aligned}
& U_{u} \sim U_{e}+u_{\tau} u_{01}(y), \quad y=O(1) \\
& U_{u} \sim u_{\tau}\left(T_{w} / T_{e}\right)^{1 / 2} \tilde{u}_{01}(\tilde{y}), \quad \tilde{y}=O(1)
\end{aligned}
$$

The form of the profile is shown in Figure 1 for $u_{\tau} \ll \epsilon \ll 1$. Equations (2.4) and (2.5) are [13], respectively, the 'law of the wake' and the 'law of the wall', written here for a compressible boundary layer, and are taken to be asymptotic representations valid as $u_{\tau} \rightarrow 0$, with $y$ and $\tilde{y}$ held fixed respectively. Throughout the analysis also $\epsilon \rightarrow 0$ such that $u_{\tau} / \epsilon \rightarrow 0$. In the wall layer the Reynolds stress and the viscous stress are both of the same order as the wall shear stress $\tau_{w}=O\left(u_{\tau}^{2}\right)$. Since $Y=O(\tilde{\delta})$ is extremely small, the momentum equation gives $\tau \sim \tau_{w}$. As $\tilde{y}=Y / \tilde{\delta} \rightarrow \infty$, the viscous stress becomes extremely small, while $\tau$ remains equal to $\tau_{w}$ in the limit, provided that also $y=Y / \delta \rightarrow 0$. The mixing length approximation $\kappa^{2} \rho\left(\tilde{y} d U_{u} / d \tilde{y}\right)^{2}=\tau_{w}+\cdots$ is introduced here for $y \ll 1$ and $\tilde{y} \gg 1$, where $\kappa$ is the von Kármán constant, taken equal to 0.41 . For a perfect gas with uniform total enthalpy, $\rho T=\rho_{w} T_{w}$ and $T=\frac{1}{2}(\gamma+1)-\frac{1}{2}(\gamma-1) U^{2}$. Integration gives, for $y \ll 1$ and $\tilde{y} \gg 1$,

$$
U_{u}=\Gamma \sin \left\{\Gamma^{-1}\left(T_{w} / T_{e}\right)^{1 / 2} u_{\tau}\left(\kappa^{-1} \ln \tilde{y}+c\right)\right\}
$$

where $c=$ constant and $\Gamma=(\gamma+1)^{1 / 2} /(\gamma-1)^{1 / 2}$. This is van Driest's [22] result, with the added simplifying assumption of uniform total enthalpy.

Expansions of Eqn. (2.6) for $U_{u} \rightarrow 1+\epsilon$ and for $U_{u} \rightarrow 0$ should agree, respectively, with expansions of the defect-layer velocity (2.4) as $y \rightarrow 0$ and of the wall-layer 


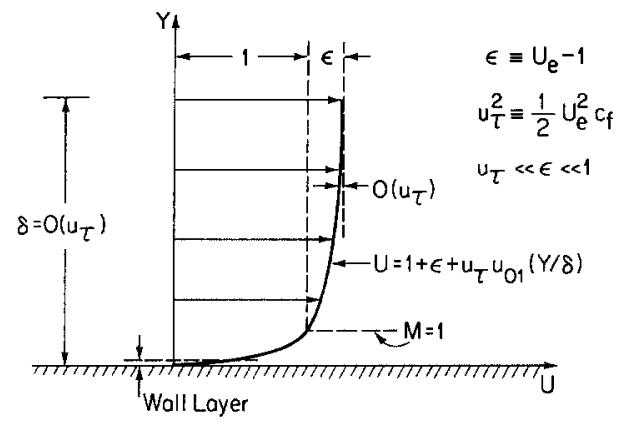

Figure 1

Undisturbed velocity profile.

velocity (2.5) as $\tilde{y} \rightarrow \infty$. For $U_{u} \rightarrow 1+\epsilon$ and $U_{u} \rightarrow 0$, respectively, Eqn. (2.6) gives

$$
\begin{aligned}
U_{u} & \sim 1+\epsilon+\left(u_{\tau} / \kappa\right)(\ln y-2 \Pi) \\
U_{u} & \sim u_{\tau}\left(T_{w} / T_{e}\right)^{1 / 2}\left(\kappa^{-1} \ln \tilde{y}+c\right)
\end{aligned}
$$

where $\Pi$ is Coles' [13] profile parameter; $c \approx 5.0$ and, for zero pressure gradient, $\Pi \approx 0.5$ or perhaps a little larger. Since $\tilde{y}=(\delta / \tilde{\delta}) y$, comparison of Eqns. (2.6) and (2.7) gives

$$
u_{\tau} \kappa^{-1} \ln (\delta / \delta)=\left(T_{e} / T_{w}\right)^{1 / 2} U_{i}(\epsilon)-u_{\tau}\left(2 \Pi_{\kappa}{ }^{-1}+c\right)
$$

where $U_{i}(\epsilon)=\Gamma \sin ^{-1}\left(\Gamma^{-1} U_{e}\right)$. The expansions (2.7) and (2.8) require, respectively, $y \rightarrow 0$ slowly and $\tilde{y} \rightarrow \infty$ slowly as $u_{\tau} \rightarrow 0$; since $u_{\tau}=O(1 / \mathrm{ln}$ Re), from Eqn. (2.9), one might take, e.g., $y=O\left(u_{\tau}^{m}\right)$ and $\tilde{y}=O\left(u_{\tau}^{-n}\right)$ as $u_{\tau} \rightarrow 0$, where $m>0$ and $n>0$. A difference from the incompressible case arises because Eqn. (2.8) with $\tilde{y}=(\delta / \tilde{\delta}) y$ does not agree with Eqn. (2.7). That is, the expansion as $\tilde{y} \rightarrow \infty$ of the wall-layer solution does not agree with the expansion as $y \rightarrow 0$ of the defect-layer solution. Thus these solutions have no common domain of validity and cannot be matched. This type of problem has been discussed in detail by Lagerstrom and Casten [23], with a model example related to flow at low Reynolds number. In the present case, the density has different values for $y=O(1)$ and for $\tilde{y}=O(1)$, and the difficulty is resolved by use of the solution (2.6) for $\tilde{\delta} \ll Y \ll \delta$; this feature was also noted by Adamson and Feo [3] and by Melnik and Grossman [4].

The defect layer, where $y=O(1)$, has nearly constant density and is described in a first approximation by incompressible-flow equations. The domain of validity of Eqn. (2.6) can be made to include $y=O(1)$ if $\kappa^{-1}(\ln y-2 \Pi)$ is replaced by $u_{01}(y)$, where $u_{01}(y)$ is the same function as for incompressible flow. Then

$$
U_{u}=\Gamma \sin \left\{\sin ^{-1}\left(\Gamma^{-1} U_{e}\right)+\Gamma^{-1}\left(T_{w} / T_{e}\right)^{1 / 2} u_{\tau} u_{01}(y)\right\}
$$

Expansion for $u_{\tau} \rightarrow 0$ gives Eqn. (2.4) if $y$ is held fixed, Eqn. (2.7) if $y \rightarrow 0$ sufficiently slowly that also $u_{\tau} \ln y \rightarrow 0$, and Eqn. (2.6) if $y=(\delta / \tilde{\delta}) \tilde{y} \rightarrow 0$ more rapidly, such that $u_{\tau} \ln \tilde{y}$ is held fixed. The use of Eqn. (2.10) was suggested by Maise and McDonald [24], 
who showed that this assumed profile permits good correlation with experimental data for adiabatic flat-plate boundary layers. Their interpretation of Eqn. (2.10) notes that a transformed velocity $\Gamma \sin ^{-1}\left(\Gamma^{-1} U_{u}\right)$ is predicted to have the incompressible form $U_{i}(\epsilon)+\left(T_{w} / T_{e}\right)^{1 / 2} u_{\tau} u_{01}(y)$ everywhere outside the wall layer.

A second relation between $\delta$ and $u_{\tau}$ for $\partial P / \partial X=0$ can be found with the help of the von Kármán integral of the momentum equation, following a derivation similar to that for incompressible flow given, e.g., by Cebeci and Smith [25]. The result is, to second order in $u_{z} / U_{e}$,

$$
\frac{m_{1}}{8} \delta=\frac{u_{\tau}}{U_{e}}+\left\{\frac{2}{\kappa} \frac{U_{e}}{U_{i}}\left(\frac{T_{w}}{T_{e}}\right)^{1 / 2}+\frac{4}{m_{1}}\left(3 \frac{T_{w}}{T_{e}}-1\right) \int_{0}^{\infty} u_{01}^{2} d y\right\} \frac{u_{\tau}^{2}}{U_{e}^{2}}
$$

The positive constant $m_{1}$ is defined by

$$
m_{1}=-8 \int_{0}^{\infty} u_{01}(y) d y
$$

and occurs in another context in the following section. For analytical purposes, the function $u_{01}(y)$ is represented in Coles' [13] form

$$
u_{01}(y)=\kappa^{-1} \ln y-\Pi \kappa^{-1}(1+\cos \pi y)
$$

for $0<y<1$, with $u_{01}(y)=0$ for $y>1$.

In the derivation which follows, the boundary-layer thickness is taken as one of two important characteristic lengths. The other length is the distance from the wall to the sonic line in the undisturbed boundary layer, denoted in nondimensional form by $\delta_{*}$. Substituting Eqn. (2.13) in Eqn. (2.10), setting $y=\delta_{*} / \delta$, and expanding for $\delta_{*} / \delta \rightarrow 0$ gives

$$
u_{\tau} \kappa^{-1} \ln \left(\delta / \delta_{*}\right)=\left(T_{e} / T_{w}\right)^{1 / 2}\left[U_{i}(\epsilon)-U_{i}(0)\right]-2 \Pi \kappa^{-1} u_{\tau} .
$$

As $\epsilon \rightarrow 0, \ln \left(\delta / \delta_{*}\right) \sim \kappa U_{\tau}^{-1} \epsilon[1-(\gamma-1) \epsilon / 4+\cdots]-2 \Pi ;$ thus $\delta_{*} / \delta \rightarrow 0$ if $u_{\tau} / \epsilon \rightarrow 0$. An alternative form of the velocity profile (2.6) in terms of a coordinate $y^{*}=Y / \delta_{*}$, is

$$
U_{u}=\Gamma \sin \left\{\sin ^{-1}\left(\Gamma^{-1}\right)+\Gamma^{-1}\left(T_{w} / T_{e}\right)^{1 / 2} \kappa^{-1} u_{\tau} \ln y^{*}\right\}
$$

for $y \rightarrow 0$ and $\tilde{y} \rightarrow \infty$.

\section{Interaction Along a Plane Wall}

As $u_{\tau} \rightarrow 0$, the orders of magnitude of the mean pressure gradient and fluid acceleration near the shock wave are larger than in the undisturbed boundary layer. The Reynolds-stress transport equations can be used to show that in most of the boundary layer the contributions to the mean forces resulting from changes in the turbulent stresses are sufficiently small, in comparison with the pressure and inertia terms, that they may be neglected as $u_{\tau} \rightarrow 0$, not only in a first approximation but also 
in the calculation of some higher-order terms. Correct asymptotic representations of the mean velocity and pressure perturbations can therefore be derived using inviscidflow equations. Also, as noted at the end of this section, displacement effects resulting from flow changes very close to the wall are extremely small, and so the largest terms in the solution for $V$ should approach zero as the distance from the wall decreases.

In the equations which follow, all laminar and turbulent stresses are neglected, as are the entropy changes across the shock wave; order-of-magnitude estimates given at the end of this section show that the neglected terms are in fact of higher order than any of the terms retained. The equations describing the fluid motion can then be written in the following form:

$$
\begin{aligned}
& a^{2} \operatorname{div} \mathbf{q}=\mathbf{q} \cdot \boldsymbol{\nabla} \frac{q^{2}}{2} \\
& a^{2}=\frac{1}{2}(\gamma+1)-\frac{1}{2}(\gamma-1) q^{2} \\
& \rho \mathbf{q} \cdot \nabla \mathbf{q}=-\gamma^{-1} \nabla P
\end{aligned}
$$

Here $\mathbf{q}, q$, and $a=(P / \rho)^{1 / 2}$ are, respectively, the velocity vector, the magnitude of the velocity, and the sound speed, all nondimensional with the critical sound speed in the external flow just ahead of the shock wave. The gradient and divergence operators imply differentiation with respect to the nondimensional variables $X$ and $Y$. Crocco's theorem, simplified by the assumption of uniform total enthalpy, is

$$
\Omega \times \mathbf{q}=\gamma^{-1} T \nabla s
$$

where $\boldsymbol{\Omega}=$ curl $\mathbf{q}$, and the specific entropy $s$ has been made nondimensional with the gas constant $R$. Since the upstream value of $V$ contributes terms of higher order than those to be retained here, the shock-polar equation beomes

$$
V_{d}^{2}=\left(U_{u}-U_{d}\right)^{2} \frac{U_{u} U_{d}-1}{2 U_{u}^{2} /(\gamma+1)-\left(U_{u} U_{d}-1\right)}
$$

where the subscripts $u$ and $d$ here denote, respectively, values immediately upstream and downstream of the shock wave. Since the jump in the velocity vector across a shock wave is in a direction normal to the shock, the shock-wave slope is

$$
\frac{d X_{s}}{d Y}=\frac{V_{d}}{U_{u}-U_{d}}
$$

where the shock-wave location is denoted by $X=X_{s}(Y)$.

If the nondimensional friction velocity $u_{\tau}$ is small in comparison with the nondimensional shock-wave strength $\epsilon$, the sonic line in the undisturbed boundary layer is very close to the wall, as can be seen from Eqn. (2.14) and Figure 1. That is, if $u_{\tau} \rightarrow 0$ and $u_{\tau} / \epsilon \rightarrow 0$, then also $\delta_{*} / \delta \rightarrow 0$. A complete description of the local pressure changes would require both an 'outer' solution, obtained by taking a limit of the equations with coordinates $Y / \delta$ and $X / \Delta$ held fixed, and an 'inner' solution, obtained with $Y / \delta_{*}$ 
Figure 2

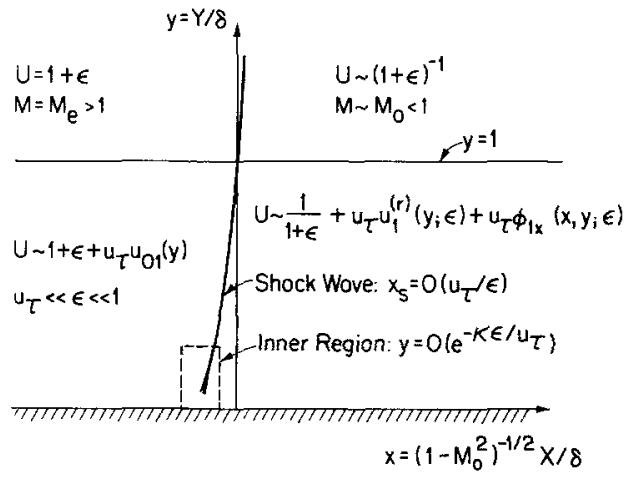

and $X / \Delta_{*}$ fixed, for suitable choices of $\Delta$ and $\Delta_{*}$. The shock wave can extend nearly to the wall, as shown in Figure 2, and so the upstream influence described by the inner solution is very small; it is shown later that $\Delta_{*}=O\left(u_{\tau}^{1 / 2} \delta_{*}\right)$, where $\delta_{*} / \delta=$ $O\left(\exp \left(-\kappa \epsilon / u_{\tau}\right)\right)$ from Eqn. (2.14). For the outer solution, therefore, $U_{u}$ can be taken equal to the undisturbed velocity (2.10) or (2.4). The inner solution describes perturbations about the undisturbed boundary-layer flow, while the outer solution describes perturbations about a different boundary-layer flow, downstream of the shock wave; the two solutions should match in a proper asymptotic sense.

For $Y=O(\delta)$ the length scale $\Delta$ in the downstream direction is found from Eqn. (3.1) and the vorticity equation to be $\Delta=O\left(b_{0} \delta\right)$, where $b_{0}^{2}(\epsilon)=1-M_{0}^{2}$ and $M_{0}$ is the Mach number in the external flow behind a normal shock wave. Coordinates $x$ and $y$ are defined by

$$
x=\frac{X}{b_{o} \delta} \quad y=\frac{Y}{\delta}
$$

where

$$
b_{o}=(\gamma+1)^{1 / 2} \epsilon^{1 / 2}\left\{1-\frac{1}{4}(2 \gamma+1) \varepsilon+\cdots\right\}
$$

Since the shock wave is nearly normal, the shock-polar equation (3.5) gives $U_{d}=$ $(1+\epsilon)^{-1}+O\left(u_{\tau}\right)$. This result suggests that throughout the flow downstream of the shock wave $U$ should be represented as a constant value $1-\epsilon+\cdots$ plus small perturbations of order $u_{\tau}$. It is convenient to separate the rotational part, which can be calculated from Crocco's theorem (3.4), and the irrotational part, which is to be found from the solution of Eqn. (3.1) satisfying the appropriate boundary conditions. In the limit as $u_{\tau} \rightarrow 0$ with $x$ and $y$ held fixed, the velocity components are then expressed in the form

$$
\begin{aligned}
U= & (1+\epsilon)^{-1}+u_{\tau} u_{1}^{(\gamma)}(x, y ; \epsilon)+u_{\tau}^{2} u_{2}^{(r)}(x, y ; \epsilon)+\cdots \\
& +u_{\tau} \phi_{1 x}(x, y ; \epsilon)+u_{\tau}^{2} \phi_{2 x}(x, y ; \epsilon)+\cdots
\end{aligned}
$$




$$
V / b_{0}(\epsilon)=u_{\tau} \phi_{1 y}(x, y ; \epsilon)+u_{\tau}^{2} \phi_{2 y}(x, y ; \epsilon)+\cdots
$$

where the functions of $\epsilon$ shown will be expanded below for $\epsilon \rightarrow 0$.

The entropy $s$ is nearly constant along a streamline, and the equation of state gives $P=\rho T$, since changes in $\overline{\rho^{\prime} T^{\prime}}$ are of higher order than terms to be retained here. It follows that along a streamline $P T^{-\gamma /(\gamma-1)} \sim P_{e} T_{u}^{-\gamma /(\gamma-1)}$ to the order required here. Substitution of $T=a^{2}$ from Eqn. (3.2) then gives the pressure as

$$
P / P_{e}=1-\gamma\left(U-U_{u}\right)-\gamma^{2}\left(U_{u}-1\right)\left(U-U_{u}\right)+\cdots
$$

Also, Crocco's theorem gives $\Omega \sim \gamma^{-1} P d s / d \psi$, where $\psi_{Y}=\rho U, \psi_{X}=-\rho V$, and $\Omega=V_{X}-U_{Y}$, and so $\Omega / P \sim \Omega_{u} / P_{e}$ along a streamline. Substitution in the expression for $\Omega$ allows calculation of terms in the rotational part of $U$ :

$$
\begin{aligned}
& u_{1}^{(r)}=(1+2 \gamma \epsilon+\cdots) u_{01}(y) \\
& u_{2}^{(r)}=-\frac{\gamma-1}{4} u_{01}^{2}(y)+\gamma \int_{y}^{\infty} u_{01}^{\prime}(y) \phi_{1 x}(x, y) d y+\cdots
\end{aligned}
$$

Substitution of the representations (3.9) and (3.10) into Eqn. (3.1) leads to differential equations for $\phi_{1}$ and $\phi_{2}$ :

$$
\begin{aligned}
\phi_{1 x x}+\phi_{1 y y}= & 0 \\
\phi_{2 x x}+\phi_{2 y y}= & -u_{2 x}^{(r)}+(1+\epsilon)\left(M_{o} / b_{0}\right)^{2}\left(2+(\gamma-1) M_{0}^{2}\right)\left(u_{1}^{(r)}+\phi_{1 x}\right) \phi_{1 x x} \\
& +(1+\epsilon) M_{0}^{2} \phi_{1 y}\left(u_{1 y}^{(r)}+2 \phi_{1 x y}\right)
\end{aligned}
$$

Expansion of the shock-wave slope (3.6) gives, after integration, the shock-wave location $x=x_{\mathrm{s}}\left(y ; u_{\tau}, \epsilon\right)$ as

$$
x_{s}=u_{\tau} x_{s 1}(y ; \epsilon)+\cdots=\frac{u_{\tau}}{2 \epsilon}\left(1+\frac{1}{2} \epsilon+\cdots\right)\left\{\phi_{1}(0, y ; \epsilon)-\phi_{1}(0,1 ; \epsilon)\right\}+\cdots
$$

where the origin of coordinates has been chosen so that $x_{s}=0$ at $y=1$. Thus the shock wave is located at $x=0$ in a first approximation, as implied in Figure 2, and the flow properties are to be studied in the quarter-plane $x>0, y>0$. Boundary conditions at $x=0$ are found from the shock-polar equation (3.5), expanded in Taylor series about $x=0$ :

$$
\begin{aligned}
\phi_{1 x}(0, y)= & -2\{1+(\gamma-1) \epsilon+\cdots\} u_{01}(y) \\
\phi_{2 x}(0, y)= & -x_{\mathrm{s} 1}(y) \phi_{1 x x}(0, y)+\frac{1}{2 \epsilon}\left\{1-\left(\gamma-\frac{3}{2}\right) \epsilon\right\} \phi_{1 y}^{2}(0, y) \\
& -\frac{\gamma-1}{2} u_{01}^{2}(y)+\cdots
\end{aligned}
$$

It is also required that $\phi_{1 y}(x, 0)=\phi_{2 y}(x, 0)=0$ and that all disturbances approach zero as $x^{2}+y^{2} \rightarrow \infty$. 
The limiting form of $\phi_{1}$ as $\epsilon \rightarrow 0$ was first given in Ref. [8]; here a term proportional to $\epsilon$ is included. The solution is expressed in terms of a distribution of sources along the $y$-axis:

$$
\phi_{1}(x, y)=-\frac{2}{\pi}\{1+(\gamma-1) \epsilon+\cdots\} \int_{-\infty}^{\infty} u_{01}(\eta) \ln \left\{x^{2}+(y-\eta)^{2}\right\}^{1 / 2} d \eta
$$

The extended definition $u_{01}(-y)=u_{01}(y)$ gives a potential for $-\infty<y<\infty$ which is symmetric about $y=0$ and thus satisfies the boundary condition there. As $x^{2}+y^{2} \rightarrow 0$, the contribution to the complex velocity is

$$
u_{\tau}\left(\phi_{1 x}-i \phi_{1 y}\right)=-2 u_{\tau}\{1+(\gamma-1) \epsilon+\cdots\} \kappa^{-1}(\ln z-2 \Pi)+\cdots
$$

where $z=x+i y$. The pressure $P_{w}$ at the wall found from evaluation of Eqn. (3.11) as $y \rightarrow 0$ is

$$
\frac{P_{w}-P_{f}}{P_{e}}=2 \gamma u_{\tau}\{1+(2 \gamma-1) \epsilon+\cdots\} \frac{2 x}{\pi} \int_{0}^{\infty} \frac{u_{01}(\eta) d \eta}{x^{2}+\eta^{2}}+\cdots
$$

where $P_{f} / P_{e}=1+\gamma\left\{2 \epsilon+(2 \gamma-1) \epsilon^{2}+\cdots\right\}$ is the pressure ratio across a normal shock wave when the upstream speed is $U_{e}=1+\epsilon$. At larger distances, as $x^{2}+$ $y^{2} \rightarrow \infty$,

$$
u_{\tau}\left(\phi_{1 x}-i \phi_{1 y}\right)=u_{\tau}\{1+(\gamma-1) \epsilon+\cdots\}\left\{\frac{m_{1}}{2 \pi z}+\cdots\right\}
$$

where $m_{1}$ is defined by Eqn. (2.12); substitution of the approximate analytical form (2.13) gives $m_{1}=8(1+\Pi) / k$. That is, the integrated effect is that of a concentrated source having nondimensional volume strength per unit length equal to $\{1+\cdots\} m_{1} u_{\tau} \delta$. One-fourth of this fluid appears to be added to the external flow in the quadrant $x>0, y>0$. Since $d(\rho U) \sim\left(1-M_{0}^{2}\right) d U$ along a streamline downstream of the shock wave, and $1-M_{0}^{2} \sim(\gamma+1) \epsilon$, the local increase in the boundary-layer displacement thickness is $\frac{1}{4}(\gamma+1) \epsilon m_{1} u_{\tau} \delta+\cdots$, as can also be found by direct calculation. An equivalent observation was made for $\epsilon=O\left(u_{\tau}\right)$ by Melnik and Grossman [5, 6]. Perturbations in turbulent stresses contribute only a higher-order change locally; the present result does not include the further displacement effect which occurs on a larger length scale as a new equilibrium velocity profile is approached. Finally, the shock-wave shape found from Eqn. (3.16) is, for $y \rightarrow 0$,

$$
x_{s}(y)-x_{s}(0)=\frac{u_{\tau}}{2 \epsilon}\left\{1+\left(\gamma-\frac{1}{2}\right) \epsilon+\cdots\right\}\left\{\frac{\pi}{\kappa} y+\cdots\right\}
$$

and, for $y \rightarrow \infty$,

$$
\begin{aligned}
x_{s}(y)-x_{s}(0)= & \frac{u_{z}}{2 \epsilon}\left\{1+\left(\gamma-\frac{1}{2}\right) \epsilon+\cdots\right\} \\
& \times\left\{\frac{m_{1}}{2 \pi} \ln y+\frac{4}{\pi} \int_{0}^{\infty} u_{01}(\eta) \ln \eta d \eta+\cdots\right\} .
\end{aligned}
$$


For $y=Y / \delta \rightarrow \infty$, the shock-wave displacement continues to increase, and should be matched with a suitable perturbed external-flow solution evaluated as $Y \rightarrow 0$.

The solution for $\phi_{2}$ can be found in two parts. A particular solution of the differential equation (3.15) can be made to satisfy homogeneous boundary conditions $\phi_{2 x}(0, y)=\phi_{2 y}(x, 0)=0$ if sources are distributed over the entire $x, y$ plane with the source strength chosen to be an even function of both $x$ and $y$. The boundary condition (3.18) at $x=0$ is then satisfied by a distribution of sources along the $y$-axis, with strength taken to be an even function of $y$ so that $\phi_{2 y}$ remains zero at $y=0$, as in the solution for $\phi_{1}$. Of special interest is the total source strength found by carrying out the integrations as $x^{2}+y^{2} \rightarrow \infty$, with the help of integrations by parts and Eqns. (3.14) and (3.17). The pressure, correct to order $u_{\tau}^{2}\left(x^{2}+y^{2}\right)^{-1 / 2}$ as $x^{2}+y^{2} \rightarrow \infty$, and the second-order source strength $m_{2}$ are found to be

$$
\begin{aligned}
& \frac{P-P_{f}}{P_{e}}=-\gamma\left\{u_{\imath}[1+(2 \gamma-1) \epsilon+\cdots] m_{1}+u_{\tau}^{2}(1+\cdots) m_{2}+\cdots\right\} \cdot \frac{1}{2 \pi} \frac{x}{x^{2}+y^{2}} \\
& m_{2}=2(5 \gamma+9) \int_{0}^{\infty} u_{01}^{2}(y) d y-2(\gamma+1) \int_{0}^{\infty} \phi_{1 y}^{2}(0, y) d y
\end{aligned}
$$

For a constant value of $y$ such that $y \gg 1, P$ initially decreases as $x$ increases from zero, reaches a minimum at $x=y$, and then increases again. However, there is a small error at the shock wave $x=x_{s}(y)=O\left(\epsilon^{-1} u_{\tau} \ln y\right)$, because the largest term in Eqn. (3.25) is $O\left(\epsilon^{-1} u_{\tau}^{2} \ln y / y^{2}\right)$, whereas the correct first approximation is found from the shockpolar equation as $-\frac{1}{2} \gamma \epsilon^{-1} u_{\tau}^{2} m_{1}^{2} /(2 \pi y)^{2}$. If it is desired, the accuracy of Eqn. (3.25) can be improved near $x=x_{s}$ by addition of a term $-\gamma u_{t}^{2} y^{2} \phi_{2 x}(0, y) /\left(x^{2}+y^{2}\right)$ with $y^{2} \phi_{2 x}(0, y)$ approximated by its leading terms $O(\ln y)$ and $O(1)$ as $y \rightarrow \infty$; away from $x=x_{s}$ the added term is smaller than the second-order term originally shown.

As $x^{2}+y^{2} \rightarrow 0$, the perturbation velocity becomes large, and it is again clear that an inner solution is required. For the choice of origin shown in Eqn. (3.16) and in Figure 2, $x_{s}(0) \neq 0$ and so the singularity in Eqn. (3.20) is displaced from its correct location through a distance $-x_{s}(0)=O\left(u_{\tau} / \epsilon\right)$. The domain of validity near $x=0$ can be extended slightly by addition of a term $-2 u_{\tau} \kappa^{-1}(1+\cdots) \ln \left(1-x_{s}(0) / z\right)$ in Eqn. (3.20) for $u_{\imath}\left(\phi_{1 x}-i \phi_{1 y}\right)$. This is accomplished formally by taking a limit as $x \rightarrow 0$ with $\epsilon x / u_{\tau}$ held fixed and then constructing a composite solution. The correction is local, and introduces only a smaller change of order $\epsilon^{-1} u_{\tau}^{2} /|z|$ when $\left|z / x_{\mathrm{s}}(0)\right| \gg 1$. The modification is, however, necessary for matching with the inner solution. A discussion of the inner solution given in Ref. [8] is briefly reviewed here, in a slightly modified form. For $Y=O\left(\delta_{*}\right)$, the undisturbed velocity is $U_{u}=1+O\left(u_{\tau}\right)$, and the differential equations show that changes in $U$ along a streamline are also $O\left(u_{\tau}\right)$ in a distance $\Delta X=O\left(u_{\tau}^{\mathbb{I} / 2} \delta_{*}\right)$. Inner variables $x^{*}$ and $y^{*}$ and disturbance velocities $u^{*}$ and $v^{*}$ are defined by

$$
x^{*}=\frac{\left(\kappa T_{e}^{1 / 2}\right)^{1 / 2}\left[X-b_{o} \delta x_{\mathrm{s}}(0)\right]}{(\gamma+1)^{1 / 2} u_{\tau}^{1 / 2} \delta_{*}}, \quad y^{*}=\frac{Y}{\delta_{*}}
$$




$$
u^{*}=\frac{\kappa T_{e}^{1 / 2}}{u_{\tau}}(U-1), \quad v^{*}=\frac{\left(\kappa T_{e}^{1 / 2}\right)^{3 / 2} V}{(\gamma+1)^{1 / 2} u_{\tau}^{3 / 2}}
$$

where factors $\left(\kappa T_{e}^{1 / 2}\right)^{1 / 2}$ have been included for convenience. Equation (3.1) and the vorticity equation are then approximated by the transonic small-disturbance equations with prescribed vorticity:

$$
\begin{aligned}
& u^{*} \partial u^{*} / \partial x^{*}-\partial v^{*} / \partial y^{*}+\cdots=0 \\
& \partial v^{*} / \partial x^{*}-\partial u^{*} / \partial y^{*}=-1 / y^{*}+\cdots
\end{aligned}
$$

The shock-wave relations (3.5) and (3.6) become

$$
\begin{aligned}
& v_{d}^{* 2}=\frac{1}{2}\left(u_{u}^{*}-u_{d}^{*}\right)^{2}\left(u_{d}^{*}+u_{u}^{*}\right)+\cdots \\
& \frac{d x_{s}^{*}}{d y^{*}}=\frac{v_{d}^{*}}{u_{u}^{*}-u_{d}^{*}}
\end{aligned}
$$

where the subscripts $u$ and $d$ again refer to quantities immediately upstream and downstream of the shock wave and the shock-wave location is given by $x^{*}=x_{s}^{*}\left(y^{*}\right)$. As $x^{*} \rightarrow-\infty, u^{*}$ approaches the undisturbed form $u^{*} \sim \ln y^{*}$; the boundary condition at the wall is $v^{*}\left(x^{*}, 0\right)=0$; and as $x^{*} \rightarrow \infty, y^{*} \rightarrow \infty$ the solution should agree with the outer solution evaluated for $x-x_{s}(0) \rightarrow 0, y \rightarrow 0$.

Although complete solutions for $u^{*}$ and $v^{*}$ can only be obtained numerically, the asymptotic behavior is found relatively easily upstream as $x^{*} \rightarrow-\infty$ and downstream as $x^{*} \rightarrow \infty, y^{*} \rightarrow \infty$. As $x^{*} \rightarrow-\infty$, the solution has the form

$$
u^{*} \sim \ln y^{*}+e^{k x^{*}} f\left(y^{*}\right), \quad v^{*} \sim k^{-1} e^{k x^{*}} f^{\prime}\left(y^{*}\right)
$$

where $f^{\prime \prime}-\left(\ln y^{*}\right) k^{2} f=0$ subject to the conditions that $f^{\prime}(0)=0$ and that incoming disturbances be absent as $y^{*} \rightarrow \infty$; the latter implies $f^{\prime} \sim-\left(\ln y^{*}\right)^{1 / 2} k f$ as $y^{*} \rightarrow \infty$. Numerical integration gives $k=0.59$. Downstream a suitable class of intermediate limits should be studied. As $y^{*} \rightarrow \infty$, a shock wave is present and must approach the nearly normal shock wave described by the outer solution. Thus, for $y^{*} \rightarrow \infty$, since $u_{u}^{*} \sim \ln y^{*}$, Eqns. (3.31) and (3.32) give $u^{*}\left(0, y^{*}\right) \sim-\ln y^{*}$. If an intermediate variable $y_{\eta}=y^{*} / \eta\left(u_{\tau}, \epsilon\right)$ is introduced, with $1 \ll \eta\left(u_{\tau}, \epsilon\right) \ll \delta / \delta^{*}$, then $\ln y^{*} \sim \ln \eta+$ ln $y_{\eta}$, where the first term is large and constant whereas the second term is $O(1)$ and variable. In each of the differential equations (3.29) and (3.30) the two largest terms remain of the same order if $x^{*}=O(\eta \sqrt{\ln \eta})$ and $v^{*}=O(\sqrt{\ln \eta})$; then $x^{*}$ and $y^{*}\left(\ln y^{*}\right)^{1 / 2}$ are of the same order. In the limit as $x^{*} \rightarrow \infty$ and $y^{*} \rightarrow \infty$ with $x^{*} /\left(y^{*} \sqrt{\ln y^{*}}\right)$ held fixed, $\ln y^{*} \sim \ln x^{*}$ and so also $x^{*} /\left(y^{*} \sqrt{\ln y^{*}}\right) \sim x^{*} /\left(y^{*} \sqrt{\ln x^{*}}\right)$. For the derivation of higher-order terms, not to be shown here, it is convenient to make this replacement. In this limit, then, the largest terms in $u^{*}+\ln y^{*}$ and $\left(\ln x^{*}\right)^{-1 / 2} v^{*}$ can be written as functions of $x^{*} /\left(y^{*} \sqrt{\ln x^{*}}\right)$. The solutions are easily obtained and the results for $U$ and $V$ finally can be rewritten as

$$
U \sim 1+\left(\kappa T_{e}^{1 / 2}\right)^{-1} u_{\tau} \ln y^{*}-\left(\kappa T_{e}^{1 / 2}\right)^{-1} u_{\tau} \ln \left\{x^{*^{2}}\left(\ln x^{*}\right)^{-1}+y^{*^{2}}\right\}
$$




$$
V \sim\left\{(\gamma+1)\left(\kappa T_{e}^{1 / 2}\right)^{-1} u_{\tau} \ln x^{*}\right\}^{1 / 2} 2\left(\kappa T_{e}^{1 / 2}\right)^{-1} u_{\imath} \tan ^{-1}\left\{y^{*}\left(\ln x^{*}\right)^{1 / 2} / x^{*}\right\}
$$

Factors $\left(1-M^{2}\right)^{1 / 2} \sim\left\{(\gamma+1)\left(\kappa T_{e}^{1 / 2}\right)^{-1} u_{\tau} \ln x^{*}\right\}^{1 / 2}$, where $M$ is the local Mach number, appear in the locations expected for solutions of the Prandtl-Glauert equation. The flow is represented by superposition of a known rotational flow and an initially unknown irrotational flow, described in terms of perturbation velocities $U-1$ and $\left(1-M^{2}\right)^{-1 / 2} V$ which are linear in $\left(\kappa T_{e}^{1 / 2}\right)^{-1} u_{\tau}$ and are functions of variables $\left(1-M^{2}\right)^{-1 / 2}\left[X-b_{o} \delta x_{s}(0)\right] / \delta_{*}$ and $Y / \delta_{*}$. For a limit such that $\left[x-x_{s}(0)\right]^{2}+y^{2} \rightarrow 0$ sufficiently slowly, with $\left(1-M^{2}\right)^{1 / 2} y /\left[x-x_{s}(0)\right]$ held fixed, the largest terms obtained if Eqns. (3.34) and (3.35) are rewritten in the outer variables $x$ and $y$ are identical to the largest terms found from Eqns. (3.9) and (3.10), with the help of Eqn. (3.12) and a modified Eqn. (3.20) in which $z$ is replaced by $x-x_{s}(0)+i y$. Introduction of the inner solution thus removes the logarithmic singularity which appears in the outer solutions for the velocity and the pressure as $x, y \rightarrow 0$.

In the derivation of these results, terms $\partial\left(\rho \overline{U^{\prime} U^{\prime}}\right) / \partial X, \partial\left(\rho \overline{U^{\prime} V^{\prime}}\right) / \partial Y$, etc., were omitted from the momentum equation, and therefore a corresponding set of terms was omitted in Eqn. (3.1) and in the calculation of the changes in vorticity. Expressions for these quantities, and therefore also order-of-magnitude error estimates for the solutions given above, can be obtained from the Reynolds-stress transport equations [25]. The equation for $\partial\left(\rho \overline{U^{\prime} U^{\prime}}\right) / \partial X$ contains, in particular, terms proportional to $\rho \overline{U^{\prime} U^{\prime}} \partial U / \partial X$. Ahead of the shock wave $\rho \overline{U^{\prime} U^{\prime}}$ is expected to be of the same order as $\rho \overline{U^{\prime} V^{\prime}}$, of order $u_{\tau}^{2}$. Relative changes at the shock wave have been estimated [26] to be proportional to the shock-wave strength and are therefore small. Thus, $\rho \overline{U^{\prime} U^{\prime}}=$ $O\left(u_{\tau}^{2}\right)$ downstream of the shock also; since $\partial U / \partial X=O\left(\epsilon^{-1 / 2}\right)$ for $X=O\left(\epsilon^{1 / 2} u_{\tau}\right)$, the product is $O\left(u_{\tau}^{2} \epsilon^{-1 / 2}\right)$. Other terms involving velocity correlations are likewise at most $O\left(u_{x}^{2} \epsilon^{-1 / 2}\right)$. Neglected terms in the expansion of Eqn. (3.1) and the vorticity equation are also of this order, and can easily be shown to be small in comparison with any of the terms retained. Similarly, the derivative of the entropy along a mean streamline contains terms proportional to $\rho \overline{U^{\prime} V^{\prime}} \partial U / \partial Y$, etc., and therefore is small enough to be neglected in the derivations above. At the shock wave the entropy jump for $Y=O(\delta)$ contains a constant term of order $\epsilon^{3}$ and functions of $y$ which are of order $\epsilon^{2} u_{\tau}, \epsilon u_{\tau}^{2}, \cdots$. It can then be shown that these changes are also sufficiently small that $\Omega / P$ and $P T^{-\gamma /(\gamma-1)}$ remain constant along a mean streamline to the order considered here. Finally, the changes in Reynolds stresses become important in a sublayer where the perturbation in $\tau_{Y}$ is no longer negligible in comparison with the perturbation in $\rho U U_{X}$. For $X=O\left(\epsilon^{1 / 2} u_{\tau}\right)$, since $\tau=O\left(u_{\tau}^{2}\right)$ and $U_{X}=O\left(\epsilon^{-1 / 2}\right)$, the sublayer is defined by $Y=O\left(u_{\tau}^{2} \epsilon^{1 / 2}\right)$. As will be shown in detail in Part II, the relative change in $\tau$ is $O(\epsilon)$, and the new term in $U$ which contributes to a displacement effect is $O\left(\epsilon \mathcal{U}_{\tau}\right)$. From the continuity equation it follows that the corresponding term in $V$ is $O\left(\epsilon^{2} u_{r}^{2}\right)$. Thus, as $y \rightarrow 0$, the largest term in the outer solution for $V$ which satisfies a nonzero boundary condition is $O\left(\epsilon^{2} u_{\tau}^{2}\right)$, smaller than any of the terms retained above. All of the neglected 
terms arising from these effects are smaller than the terms retained by at least a factor of order $\epsilon$.

\section{Geometric Effects and Comparison with Experiment}

The theory of the preceding section leads to a limiting form for the pressure distribution as $u_{\tau} \rightarrow 0$ and $u_{\tau} / \epsilon \rightarrow 0$, for unseparated flow. In the flow past an airfoil at supercritical speed, with a shock wave terminating a region of supersonic flow, the additional effect of surface curvature can also be important in changing the pressure distribution and delaying separation, as discussed below and in Part II. The boundary layer might remain attached for $M_{e}$ up to about 1.25, depending on the profile shape; Re may be about $5 \times 10^{7}$ or perhaps as high as $10^{8}$; and the flow ahead of the shock wave experiences a favorable pressure gradient, with magnitude which depends on the airfoil shape, so that the profile parameter $\Pi$ is smaller than 0.5 (e.g., Ref. [30]). For a combination of parameters which is favorable with regard to requirements of the present theory, with $M_{e}=1.26, \operatorname{Re}=10^{8}$, and $\Pi=0$, the relative position of the sonic line is given by $\delta_{*} / \delta=0.10$. This value would increase as $M_{e}$ or Re decreases or as $\Pi$ increases, as seen from Eqn. (2.14). Experimental results, however, are not yet available with detailed local pressure measurements for values of the parameters which correspond to such airfoil flows and which meet the requirements of the theory. For all available data, either the flow is separated or the values of the parameters are such that the sonic line is not close to the wall. Nonetheless, a comparison with data from Refs. [1] and [2] has been carried out, and the agreement seems favorable provided that corrections for geometric effects are included.

A wall having convex longitudinal curvature is described locally by $Y \sim-\frac{1}{2} K X^{2}$, where $K \ll 1$ if the radius of curvature is large in comparison with the reference length used in the definitions of $X$ and $Y$. A local solution for the inviscid external flow near the foot of a normal shock wave shows a discontinuity in streamline curvature [27, 28]. Ahead of the shock wave $P_{Y}>0$ to provide the required acceleration toward the wall; if the flow is irrotational, it follows that $U_{Y}<0$. The shock-wave relations give $U_{Y}>0$ and $P_{Y}<0$ downstream; therefore also $V_{X}>0$, whereas the tangency condition at the wall requires $V_{X}<0$ as $Y \rightarrow 0$. The term in the complex velocity which satisfies the required conditions as $X, Y \rightarrow 0$ has the derivative

$$
b_{0} U_{X}^{(c)}-i V_{X}^{(c)} \sim-(4 / \pi) K \ln Z+i K+O(K)
$$

for $0 \leq \arg Z \leq \frac{1}{2} \pi$, where $Z=b_{0}^{-1} X+i Y$ and, as before, $b_{0}^{2}(\epsilon) \sim(\gamma+1) \epsilon$. The largest omitted term is of order $K$ and is real; the value depends on the flow description for $Z=O(1)$, and is known for symmetric two-dimensional or axisymmetric nozzle flows [29].

Terms $U^{(c)}$ and $V^{(c)}$, of order $K \epsilon^{-1 / 2} u_{\tau} \ln u_{\tau}$ and $K u_{\tau}$ respectively when $Z=O\left(u_{\tau}\right)$, are now added to the expansions of $U$ and $V$ given by Eqns. (3.9) and (3.10). The rotational part of $U$ is unchanged, and reformulation of the boundary-value problem 
Figure 3

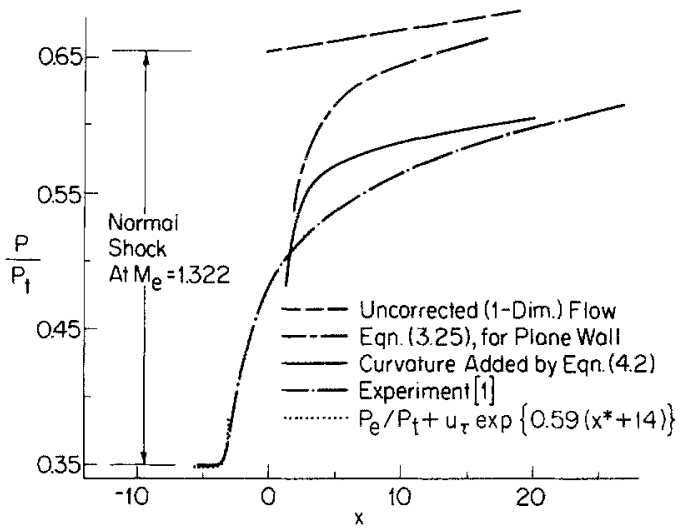

Pressure at wall with longitudinal curvature: $M_{\varepsilon}=1.322, \mathrm{Re}=9.6 \times 10^{5}, K \approx 0.2$.

for the perturbation potential shows that $\phi_{1}$ is unchanged, whereas now $\phi_{2}$ depends on $K$, through nonlinear terms in the potential equation; that is, $\phi_{2}=\phi_{2}(x, y ; \epsilon, K)$. The new terms in $\phi_{2}$ contribute a change in $U$ which is $O\left(K u_{\tau}^{2}\right)$, smaller than terms retained previously provided that $K=o(1)$. Thus, to the order considered here, for $u_{\tau} / \epsilon \rightarrow 0$, a curvature correction is simply added to the earlier results. The new term in the pressure, written in terms of $x$ and $y$, is

$$
P^{(c)}=\frac{2 \gamma K \delta}{\pi}\left\{2 x \ln \delta+x \ln \left(x^{2}+y^{2}\right)+\frac{\pi}{2} y-2 y \tan ^{-1} \frac{y}{x}+A x\right\}
$$

where the constant $A$ is determined only if a solution is known for the external flow at larger distances.

An early careful and comprehensive experimental study was carried out by Ackeret, Feldmann, and Rott [1]. In Figures 3 and 4, predicted pressures are compared with their experimental results for $M_{e}=1.32$, corresponding to $\epsilon=0.247$, and

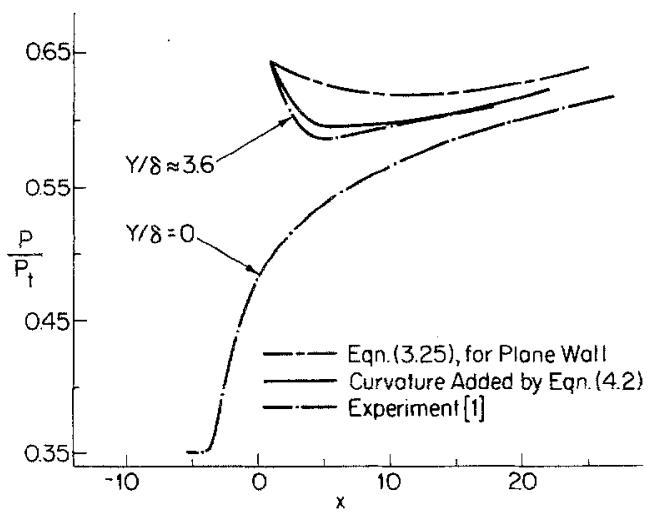

Figure 4

Pressure outside boundary layer, at $Y / 8 \approx 3.6$, for curved wall: $M_{e}=1.322, \operatorname{Re}=9.6 \times 10^{5}$, $K \approx 0.2$. 
$\operatorname{Re}=9.6 \times 10^{5}$, based on distance to the shock wave. Equations (2.9), (2.11) and (2.14) are used for approximate evaluation of other parameters. One more experimental value is needed; $\delta_{*}$ is chosen since it is easily read from the measured velocity profile and since only $\ln \delta_{*}$ enters the equations, so that an error has small effects on other quantities. For $\delta_{*}=0.0055$, the calculations give $u_{\tau}=0.051, \delta=0.021$, and $\Pi=0.28$. This value of $\Pi$ seems plausible (e.g., Ref. [30]) because of the observed small favorable pressure gradient ahead of the shock wave. An adverse gradient of about the same magnitude is evident downstream, and is estimated here by $P_{t}^{-1} \partial P / \partial X \approx 0.12$, where $P_{t}$ is the upstream stagnation pressure. For the 'uncorrected' curve in Figure 3, the flow is imagined to be the same as a one-dimensional channel flow with this pressure gradient. In Eqn. (4.2) a corresponding term is added, and the term proportional to $K \delta A x$ is neglected. The local curvature of the plate can be inferred from measured pressures immediately behind the shock wave. It is estimated that $P_{t}^{-1} \partial P / \partial Y \approx 0.15$; since $P_{Y} \sim-\gamma V_{X}$, it follows that $K \approx 0.2$. With the kind of assistance of Prof. $Z$. Plaskowski of the Institut für Aerodynamik, ETH Zürich, the author was able to measure ordinates of the plate actually used in the experiments; values in an appropriate neighborhood confirm the estimate $K \approx 0.2$. The origin $x=0$ is chosen at the estimated position of the shock wave at the edge of the boundary layer, found using measured pressures outside the boundary layer together with Eqn. (3.24).

The comparison in Figures 3 and 4 shows that the curvature effect is comparable in importance with the boundary-layer displacement effect; addition of the curvature term leads to a more pronounced 'shoulder' in the predicted wall pressure distribution. The longitudinal pressure gradient due to tunnel divergence is also seen to be important. At the plate for typical values of $x$, say $4<x<14$, the prediction gives about 75 percent of the pressure drop below the value for a one-dimensional flow; outside the boundary layer, at $Y / \delta \approx 3.6$, the agreement is somewhat better. It is found that the velocity in Eqn. (3.21) is closely approximated by (const.)/ $x$ for $x \lesssim 2$, so that Eqn. (3.25) for the pressure is adequate here, with the correction (4.2). Modest changes in the assumed values of the parameters do not have a major effect on the comparison; for example, at a given $X, m_{1} / x$ does not depend strongly on $\Pi$ because $\delta$ increases if $\Pi$ decreases. The upstream exponential decay predicted by Eqn. (3.33) is also shown in Figure 3, in the form $\Delta P / P_{t} \sim u_{\tau} \exp \left\{k\left(x^{*}-x_{0}^{*}\right)\right\}$, with $x_{0}^{*}$ taken equal to -14 for approximate agreement with experiment. A major difficulty with this comparison is that the upstream sonic line lies at about $y=\delta_{*} / \delta=0.26$, and the shock wave ends at a still larger distance from the wall, so that the inner region for $x^{*}=O(1), y^{*}=O(1)$ is not negligibly small. At a higher Reynolds number and therefore a lower $u_{\tau}$, the shock wave would extend closer to the wall, and the size of the region in Figure 3 where no prediction is given would be smaller. A second serious difficulty arises because the flow probably was separated. The authors of Ref. [1] stated that reversed flow could not be ascertained at any point; however, the velocity profiles shown seem inconclusive, since measurements were not possible very close to the wall. Calculations based on the theory of Part II of the present paper, for the parameter values given in 
Ref. [1], suggest that the flow was in fact separated, with a very thin separation bubble having length equal to a few boundary-layer thicknesses. The effect of such a bubble would give a more gradual pressure rise in the region of greatest disagreement in Figure 3. Finally, a slight unsteadiness in the shock wave position would also contribute to a decrease in the measured pressure gradient.

For a comparison of predicted pressures with the data of Ref. [2], a correction for flow in a circular pipe should be added. Cylindrical coordinates $x^{+}$and $r^{+}$are defined by

$$
x^{+}=\frac{X}{b_{o}(\epsilon) R}=\frac{\delta}{R} x, \quad r^{+}=1-\frac{Y}{R}=1-\frac{\delta}{R} y
$$

where $R$ is the ratio of the local pipe radius to the reference length, and $Y$ is measured inward from the wall, so that $r^{+}=0$ at the axis. Solutions are to be found for $\epsilon \rightarrow 0$, $u_{\tau} / \epsilon \rightarrow 0$ with $x^{+}, r^{+}$fixed. The wall shape is given by $r^{+}=1+\epsilon^{2} f(X / R)$ with $f=0$ at $X / R=0$. Velocity components $U^{+}, V^{+}$in the $x^{+}, r^{+}$directions can be written with the local curvature and boundary-layer effects shown separately:

$$
\begin{aligned}
& U^{+}=U^{(0)}\left(X \mid R, r^{+} ; \epsilon\right)+K U^{(1)}\left(x^{+}, r^{+}\right)+\frac{1}{2} m_{1} u_{\tau} \frac{\delta}{R}+u_{\tau} \frac{\delta}{R} U^{(2)}\left(x^{+}, r^{+}\right)+\cdots \\
& V^{+}=V^{(0)}\left(X \mid R, r^{+} ; \epsilon\right)+K b_{o}(\epsilon) V^{(1)}\left(x^{+}, r^{+}\right)+u_{\tau} \frac{\delta}{R} b_{o}(\epsilon) V^{(2)}\left(x^{+}, r^{+}\right)+\cdots
\end{aligned}
$$

where now $K=\epsilon^{2} f^{\prime \prime}(0)$ is the wall curvature at the foot of the shock wave, made nondimensional with the reciprocal of the pipe radius. The terms $U^{(0)}$ and $V^{(0)}$ are the terms which would be present if the effects of the shock wave were ignored [29]. Terms proportional to $K$ contain the local curvature effect, and terms proportional to $u_{\tau} \delta / R$ contain the local boundary-layer displacement effect. The latter is described in terms of a ring source of radius $r^{+}=1$ located at $x^{+}=0$ and having volume strength per unit length equal to $m_{1} u_{\tau} \delta+\cdots$; numerical solutions for $u_{\tau} / \epsilon=O(1)$ given by Melnik and Grossman [6] also include this effect. For $x^{+} \rightarrow \infty$, the fluid added at the source gives an increase of $\frac{1}{2} m_{1} u_{\tau} \delta / R$ in $U^{+}$, shown explicitly in Eqn. (4.4).

The local solutions for $X / R=O\left(\epsilon^{1 / 2}\right)$ are found in terms of a stream function defined by $\partial \psi^{(i)} / \partial r^{+}=r^{+} U^{(i)}, \partial \psi^{(i)} / \partial x^{+}=-r^{+} V^{(i)}$, where $i=1$, 2. The largest terms in Eqn. (3.1), combined with the irrotationality condition, lead finally to

$$
\psi^{(i)}\left(x^{+}, r^{+}\right)=\sum_{n=1}^{\infty} a_{n}^{(i)} e^{-\lambda_{n} x^{+}} r^{+} J_{1}\left(\lambda_{n} r^{+}\right)
$$

where $J_{1}\left(\lambda_{n}\right)=0$ for $n=1,2,3, \ldots$, so that the wall boundary condition $\psi^{(i)}\left(x^{+}, 1\right)=0$ is satisfied; also $\psi^{(i)} \rightarrow 0$ as $x^{+} \rightarrow \infty$, and boundary values are to be specified at $x^{+}=0$. To the order required, the shock-polar equation reduces to the Prandtl 
relation, and so $\psi^{(1)}=r^{+2}\left(1-r^{+2}\right) / 4$ at $x^{+}=0$. The condition that the ring source gives no term of order $u_{\tau} \delta / R$ in $U^{+}$at $x^{+}=0$ implies $\psi^{(2)}=-m_{1} r^{+2} / 4$ at $x^{+}=0$. Comparison with the wall boundary condition shows that $\psi^{(2)}$ is discontinuous at the foot of the shock wave $x^{+}=0, r^{+}=1$; the value obtained as $x^{+} \rightarrow 0, r^{+} \rightarrow 1$ depends on the direction of approach. The coefficients $a_{n \text {. }}^{(1)}$ can be found from the solutions of Messiter and Adamson [29] or by direct calculation, and the coefficients $a_{n}^{(2)}$ are found directly:

$$
a_{n}^{(1)}=-\frac{4}{\lambda_{n}^{3} J_{o}\left(\lambda_{n}\right)} \quad a_{n}^{(2)}=\frac{m_{1}}{2 \lambda_{n} J_{o}\left(\lambda_{n}\right)}
$$

for $n=1,2,3 \ldots$

For calculation of the pressure distribution and the shock-wave shape, it is convenient to introduce the corresponding velocity potential $\phi^{(2)}$, which satisfies $\partial \phi^{(2)} / \partial x^{+}=U^{(2)}$ and $\partial \phi^{(2)} / \partial r^{+}=V^{(2)}$, and which has a logarithmic singularity at $x^{+}=0, r^{+}=1$. With the help of the asymptotic form for $J_{o}\left(\lambda_{n} r^{+}\right)$, one can show the singular part explicitly:

$$
\begin{aligned}
& \phi^{(2)}\left(x^{+}, r^{+}\right)=-\frac{m_{1}}{2} \sum_{n=1}^{\infty}\{\left\{\frac{1}{\lambda_{n}} \frac{J_{o}\left(\lambda_{n} r^{+}\right)}{J_{0}\left(\lambda_{n}\right)} e^{-\lambda_{n} x^{+}}-\frac{(-1)^{n}}{\pi\left(n+\frac{1}{4}\right)\left(r^{+}\right)^{1 / 2}}\right. \\
&\left.\times e^{-\pi(n+1 / 4) x^{+}} \cos \left[\pi\left(n+\frac{1}{4}\right) r^{+}-\frac{\pi}{4}\right]\right\} \\
&-\frac{m_{1}}{2 \pi\left(r^{+}\right)^{1 / 2}} \mathscr{R}\left\{\ln \frac{1+\zeta}{1-\zeta}+2 \tan ^{-1} \zeta-4 \zeta\right\}
\end{aligned}
$$

where $\ln \zeta=-(\pi / 4)\left\{x^{+}+i\left(1-r^{+}\right)\right\}$, and $\mathscr{R}$ indicates that the real part is to be taken. As $x^{+} \rightarrow 0$ and $r^{+} \rightarrow 1$, the largest term in the complex velocity $U^{(2)}-i V^{(2)}$ is due to a two-dimensional source of strength $m_{1}$, in agreement with Eqn. (3.22). The change in the boundary-layer displacement effect is then found by subtracting the source term from $U^{(2)}-i V^{(2)}$ and adding the constant term which remains as $x^{+} \rightarrow \infty$. If the numerically small contribution of the infinite series is omitted, the corresponding correction to the wall pressure is

$$
\frac{\Delta P_{w}}{P_{e}}=-\gamma u_{\imath} \frac{\delta}{R} \frac{m_{1}}{2}\left(1+\frac{e^{-5 \pi x^{+} / 4}}{1-e^{-\pi x^{+}}}-\frac{1}{\pi x^{\ddagger}}\right)
$$

As $x^{+}=X /\left(b_{0} R\right) \rightarrow 0, \Delta P_{w} / P_{e}$ approaches a constant value $-\frac{1}{8} \gamma m_{1} u_{\tau} \delta / R$, which implies an additional second-order correction to the boundary-layer solution found in Section 3 for $X=O\left(b_{0} \delta\right)$. The shock-wave shape is found directly from the potential; in particular, as $r^{+} \rightarrow 1$ the displacement of the shock from its intersection with the axis is found by adding the perturbation potential from Section 3 to that found here, and subtracting the common term proportional to $\ln Y$. The result is

$$
\Delta X_{S}=-(4 \pi \epsilon)^{-1}(\gamma+1)^{1 / 2} \epsilon^{1 / 2} m_{1} u_{\tau} \delta \ln (R / \delta)+O\left(u_{\tau} \delta \epsilon^{-1 / 2}\right)
$$




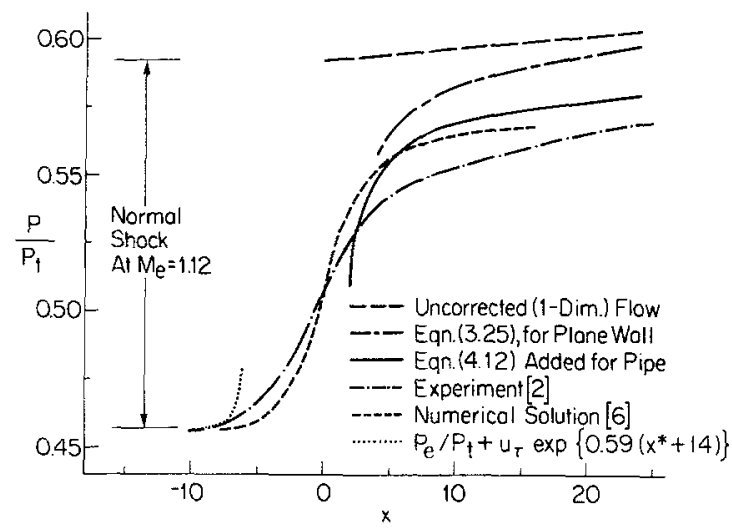

Figure 5

Pressure at wall of circular pipe: $M_{e}=1.12, \operatorname{Re} \approx 6 \times 10^{6}, \delta / R \approx 0.055$.

In Figure 5 a comparison is made with pressures measured in a circular pipe by Gadd [2], for $M_{e}=1.12$ and $\epsilon=0.097$. The length of an equivalent flat-plate boundary layer is not a given quantity; instead, Gadd's estimated value for boundary-layer thickness is used here, along with the estimate $\delta_{*} / \delta=0.45$ found from the measured velocity profile. The sonic line is therefore still further from the wall than in the Ackeret experiment. Other approximate values are calculated as $u_{\tau}=0.04, \delta=0.02$, and $\Pi=0.1$; the Reynolds number corresponding to these values is $\operatorname{Re}=6 \times 10^{6}$. A pressure gradient due to small divergence of the test section is estimated downstream by $P_{t}^{-1} \partial P / \partial X=0.06$. The effect of finite pipe radius is seen to be about as large as the boundary-layer displacement effect. The upstream exponential decay is also shown, with $x_{0}^{*}$ again taken equal to -14 . Again there is a relatively large region where no prediction is made and where numerical solution of the transonic small-disturbance equations is required. Such a solution was obtained by Melnik and Grossman [4] for this case and is also shown in Figure 5. For large $x$ the analytical and numerical solutions differ by an amount about equal to the correction for the change in pipe cross-section area.

\section{Concluding Remarks}

The interaction of a turbulent boundary layer with a weak normal shock wave has been described here and elsewhere $[3,4,8]$ in terms of rational approximations based on systematic asymptotic expansion procedures. The interaction is characterized by two small parameters, a nondimensional friction velocity $u_{\tau}$ and a nondimensional shock-wave strength $\epsilon$, and limiting forms of the local solutions can be studied as $u_{\tau} \rightarrow 0$ and $\epsilon \rightarrow 0$. For the case $u_{\tau} / \epsilon \rightarrow \infty$ [3], analytical solutions indicated that separation does not occur; solutions for $u_{\tau} / \epsilon$ held fixed [4], with the first approximation described by the transonic small-disturbance equations, gave the same result. If, finally, $u_{\tau} / \epsilon \rightarrow 0$, it appeared that analytical solutions would be possible and that 
perhaps the onset of separation could be discussed. Solutions for the pressure have been obtained here and will be used in Part II for the calculation of wall shear stress and a discussion of incipient separation.

The largest terms in the pressure, of order $u_{\tau}$, are derived quite easily, and a number of higher-order effects have been added. Corrections of order $\epsilon u_{\tau}$ give, e.g., a $35 \%$ change if $M_{e}=1.25$. A partial solution for terms of order $u_{\tau}^{2}$ shows that these terms likewise are significant, typically giving changes of $25 \%$ to $50 \%$ for $\operatorname{Re}=10^{6}$ or $10^{7}$. Corrections of order $K u_{\tau}$ and $u_{\tau} \delta / R$, obtained in analytical form for a wall with longitudinal curvature and for a circular pipe respectively, are found to be numerically important for the tests of Refs. [1] and [2].

In the solutions for these higher-order terms, the dependence on the parameters is of course shown explicitly, and the relative importance of different effects is therefore apparent. It is not, however, possible to obtain analytical solutions in the asymptotically small inner region which accounts for the upstream influence. For values of the parameters corresponding to actual transonic flight conditions, it is possible for this region to be relatively small. Experimental results, however, are not available in this parameter range; for existing data, either the flow is separated or the sonic line is not close to the wall. Nonetheless, some comparisons with such data were attempted, and the agreement seems fairly good downstream from the inner region. The predicted pressures remain somewhat higher than the experimental values, and the correction terms calculated thus far are large enough to suggest that additional higher-order terms would be likely to give still further improvement.

An essential feature of the asymptotic flow descriptions in terms of $u_{\tau}$ and $\epsilon$ is the two-layer structure of the undisturbed profile, expressed by the law of the wake and the law of the wall. It is this property which permits the calculation of interaction pressures without knowledge of changes in shear stresses close to the wall. In other studies [31,32] which were not based on use of this profile, derivation of a sublayer solution was necessary before the calculation of the pressure could be completed; these studies also introduced a linearized formulation for the main part of the boundary layer. In the present asymptotic description for $u_{\tau} / \epsilon \rightarrow 0$, sublayer effects do not appear even among the second-order terms in the pressure. Linear equations appear naturally as a consequence of the limiting case considered, and the procedure for adding higherorder terms is clear, at least up to the small order of magnitude at which changes in the Reynolds stresses must be taken into account. In the formulations for $u_{\tau} / \epsilon$ held fixed [4] and for $u_{\tau} / \epsilon \rightarrow \infty$ [3], again the flow details near the wall do not influence the pressure.

Asymptotic flow descriptions based on the two-layer undisturbed profile are therefore now available for the entire range $0<u_{\tau} / \epsilon<\infty$, with numerical solutions obtained as $u_{\tau} \rightarrow 0$ and $\epsilon \rightarrow 0$ if $u_{\tau} / \epsilon$ is fixed and analytical solutions if $u_{\tau} / \epsilon \rightarrow \infty$ or $u_{\tau} / \epsilon \rightarrow 0$. For accurate calculations in parameter ranges of practical interest, some further extensions appear to be needed. In the present case, as $u_{\tau} / \epsilon \rightarrow 0$, the necessary condition that the sonic line be close to the wall is met for a relatively narrow range 
of the parameters. The solutions for wall pressure would be more useful if a simple curve fit were introduced for the inner region, say by means of a straight line tangent to the source solution downstream and to the exponential solution upstream. The choice $x_{0}^{*} \approx-14$ in the exponential term was made for agreement with experiment in Figure 3; the results shown in Figure 5 suggest that the magnitude is too large and that perhaps a more suitable tentative value would be $x_{0}^{*}=-10$. The present solutions also suggest that terms of higher order than those retained in Ref. [4] are likely to be important for $u_{\tau} / \epsilon=O(1)$. In this case the curvature correction would no longer have a simple form, but would have to be incorporated in the numerical solution through the use of modified boundary conditions. Moreover, it appears that certain terms of order $u_{\tau}^{2}$, and possibly still other higher-order corrections, will be essential for numerical accuracy in the wall shear (Part II) as well as in the pressure. Finally, the local interaction influences the potential flow at larger distances; the manner of introducing corrections in the external fiow deserves further study.

\section{Acknowledgments}

Support for this work by NASA-Langley Research Center, under Research Grant No. NSG 1326, is gratefully acknowledged. The author wishes to thank Prof. T. C. Adamson, Jr., for his initial suggestion that the present study for $u_{t} / \epsilon \rightarrow 0$ should be undertaken, and for continuing helpful discussions concerning the work. Part of the work was carried out while the author was a visitor at the Institut für Aerodynamik, ETH Zürich; the author wishes to thank Prof. Nicholas Rott for his kind hospitality during this visit.

\section{References}

[1] J. ACKERET, F. FeLDMANN, and N. RoTr, Untersuchungen an Verdichtungsstössen und Grenzschichten in schnell bewegten Gasen, Mitteilungen aus dem Inst. für Aerodynamik, ETH Zürich, Nr. 10 (1946). Translated as NACA TM 1113 (1947).

[2] G. E. GADD, Interactions between Normal Shock Waves and Turbulent Boundary Layers, A.R.C. 22559, R\&M 3262 (1961).

[3] T. C. AdAmson, Jr. and A. Feo, Interaction between a Shock Wave and a Turbulent Boundary Layer in Transonic Flow, SIAM J. Appl. Math. 29, 121-145 (1975).

[4] R. E. Melnik and B. Grossman, Analysis of the Interaction of a Weak Normal Shock Wave with a Turbulent Boundary Layer, AIAA Paper No. 74-598 (1974).

[5] R. E. Melnik and B. Grossman, 'Further Developments in an Analysis of the Interaction of a Weak Normal Shock Wave with a Turbulent Boundary Layer', Symposium Transsonicum II, K. Oswatitsch and D. Rues, eds., Springer-Verlag, Berlin (1976), pp. 262-272.

[6] R. E. Melnik and B. Grossman, 'Interactions of Normal Shock Waves with Turbulent Boundary Layers at Transonic Speeds', Transonic Flow Problems in Turbomachinery, T. C. Adamson, Jr. and M. F. Platzer, eds., Hemisphere Publ. Co. (1977), pp. 415-433.

[7] T. C. Adamson, Jr., 'The Structure of Shock Wave-Boundary Layer Interactions in Transonic Flow', Symposium Transsonicum II, K. Oswatitsch and D. Rues, eds., Springer-Verlag, Berlin (1976), pp. 244-251.

[8] T. C. Adamson, Jr. and A. F. Messiter, "Normal Shock Wave-Turbulent Boundary Layer Interactions in Transonic Flow near Separation', Transonic Flow Problems in Turbomachinery, T. C. Adamson, Jr. and M. F. Platzer, eds., Hemisphere Publ. Co. (1977), pp. 392-414. 
[9] T. C. Adamson, Jr. and A. F. Messirer, Shock Wave-Turbulent Boundary Layer Interactions in Transonic Flow, Adv. in Engineering Sci., NASA CP-2001 (1976), pp. 1425-1435.

[10] A. F. Messiter and T. C. Adamson, Jr., A Study of the Interaction of a Normal Shock Wave with a Turbulent Boundary Layer at Transonic Speeds, Advanced Technology Airfoil Research, NASA CP-2045 (1978), vol. I, pp. 271-279.

[11] T. VON KÁRMÁN, Theorie des Reibungswiderstandes, Konferenz über Hydromechanische Probleme des Schiffsantriebs, Hamburg, 1932. Reprinted in Collected Works of Theodore von Kármán, Butterworths, London (1956), vol. II, pp. 394-414.

[12] C. B. Millikan, A Critical Discussion of Turbulent Flows in Channels and Circular Tubes, Proc. 5th Int. Cong. Appl. Mech. (1938), pp. 386-392.

[13] D. E. Coles, The Law of the Wake in the Turbulent Boundary Layer, J. Fluid Mech. 1, 191-226 (1956).

[14] H. TEnNekes, Outline of a Second-Order Theory of Turbulent Pipe Flow, AIAA J. 6, 1735-1740 (1968).

[15] K. S. YAJNIK, Asymptotic Theory of Turbulent Shear Flows, J. Fluid Mech. 42, 411-427 (1970).

[16] G. L. MelLoR, The Large Reynolds Number Asymptotic Theory of Turbulent Boundary Layers, Int. J. Eng. Sci. 10, 851-873 (1972).

[17] W. B. Bush and F. E. Fendell, Asymptotic Analysis of Turbulent Channel and BoundaryLayer Flow, J. Fhid Mech. 56, 657-681 (1972).

[18] N. AfZaL, A Higher Order Theory for Compressible Turbulent Boundary Layers at Moderately Large Reynolds Number, J. Fluid Mech. 57, 1-25 (1973).

[19] K. Stewartson and P. G. Williams, Self-Induced Separation, Proc. Roy. Soc. A 312, 181-206 (1969).

[20] A. F. Messiter, A. Feo, and R. E. Melnik, Shock-Wave Strength for Separation of a Laminar Boundary Layer at Transonic Speeds, AIAA J. 9, 1197-1198 (1971).

[21] H. M. Brilliant and T. C. Adamson, Jr., Shock Wave Boundary Layer Interactions in Laminar Transonic Flow, AIAA J. 12, 323-329 (1974).

[22] E. R. VAN DRIEST, Turbulent Boundary Layer in Compressible Fluids, J. Aero. Sci. 18, 145-160 (1951).

[23] P. A. Lagerstrom and R. G. Casten, Basic Concepts Underlying Singular Perturbation Techniques, SIAM Rev. 14, 63-120 (1972).

[24] G. MAISE and H. MCDonaLD, Mixing Length and Kinematic Eddy Viscosity in a Compressible Boundary Layer, AIAA J. 6, 73-80 (1968).

[25] T. CebBeci and A. M. O. Smith, Analysis of Turbulent Boundary Layers. Academic Press, New York (1974).

[26] H. S. RIBNER, Shock-Turbulence Interaction and the Generation of Noise, NACA TN 3255 (1954).

[27] G. E. GADD, The Possibility of Normal Shock Waves on a Body with Convex Surfaces in Inviscid Transonic Flow, Z. Angew. Math. Phys. 11, 51-58 (1960).

[28] K. OsWATITSCH and J. ZIEREP, Das Problem des senkrechten Stosses an einer gekrümmten Wand, Z. Angew. Math Mech. 40, 143-144 (1960).

[29] A. F. Messiter and T. C. Adamson, Jr., On the Flow near a Weak Shock Wave Downstream of a Nozzle Throat, J. Fluid Mech. 69, 97-108 (1975).

[30] J. E. Lewis, R. L. Gran, and T. Kubota, An Experiment on the Adiabatic Compressible Turbulent Boundary Layer in Adverse and Favorable Pressure Gradients, J. Fluid Mech. 51, 657-672 (1972).

[31] R. BOHNING and J. ZTEREP, Der senkrechte Verdichtungsstoss an der gekrümmien Wand unter Berücksichtigung der Reibung, Z. Angew. Math. Phys. 27, 225-240 (1976).

[32] G. R. Inger and W. H. MASON, Analytical Theory of Transonic Normal Shock-Turbulent Boundary-Layer Interactions, AIAA J. 14, 1266-1272 (1976).

\section{Summary}

Asymptotic solutions are derived for the pressure distribution in the interaction of a weak normal shock wave with a turbulent boundary layer. The undisturbed boundary layer is characterized by the law of the wall and the law of the wake for compressible flow. In the limiting case considered, for 'high' transonic speeds, the sonic line is very close to the wall. Comparisons with experiment are shown, with corrections included for the effect of longitudinal wall curvature and for the boundary-layer displacement effect in a circular pipe. 


\section{Zusammenfassung}

Asymptotische Lösungen für den Druckverlauf bei der Wechselwirkung zwischen einem schwachen normalen Stoss und einer turbulente Grenzschicht werden hergeleitet. Das Wandgesetz und Geschwindigkeitsdefekt-Gesetz für kompressible Strömung kennzeichnen die ungestörte Grenzschicht. Der Grenzfall hoher transsonischen Strömung, in dem die Schallinie in der Nähe der Wand liegt, wird untersucht. Die theoretischen Ergebnisse werden mit Experimenten verglichen. Dabei wird die Wandkrümmung und im Fall der Rohrströmung die Verdrängungsdicke berücksichtigt.

(Received: May 30, 1979) 